\title{
Progress on the Development of the $\mathrm{Nb}_{3} \mathrm{Sn}$ 11T Dipole for the High Luminosity Upgrade of LHC
}

\author{
F. Savary, M. Bajko, B. Bordini, L. Bottura, L. Fiscarelli, J. Fleiter, A. Foussat, S. Izquierdo Bermudez, \\ F. Lackner, C. H. Löffler, E. Nilsson, J.C. Perez, H. Prin, R. Principe, D. Ramos, G. de Rijk, \\ D. Smekens, S. Sequeira Tavares, G. Willering, A.V. Zlobin
}

\begin{abstract}
The High Luminosity LHC Project at CERN entered into the production phase in October 2015 after the completion of the design study phase. In the meantime, the development of the $11 \mathrm{~T}$ dipole needed for the upgrade of the collimation system of the machine made significant progress with very good performance of the first two-in-one magnet model of 2-m length made at CERN. The $11 \mathrm{~T}$ dipole, which is more powerful than the current main dipoles of LHC, can be made shorter with an equivalent integrated field. This will allow creating space for the installation of additional collimators in specific locations of the dispersion suppressor regions. Following tests carried out during heavy ions runs of $\mathrm{LHC}$ in the end of 2015 , and a more recent review of the project budget, the installation plan for the $11 \mathrm{~T}$ dipole was revised. Consequently, one $11 \mathrm{~T}$ dipole full assembly containing two $11 \mathrm{~T}$ dipoles of $5.5-\mathrm{m}$ length will be installed on either side of interaction point 7 . These two units shall be installed during the long shutdown 2 in years 2019-2020. After a brief reminder on the design features of the magnet, this paper describes the current status of the development activities, in particular the short model programme and the construction of the first full scale prototype at CERN. Critical operations like the reaction treatment and the coil impregnation are discussed, the quench performance tests results of the two-in-one model are reviewed and finally, the plan towards the production for the long shut downs 2 is described.
\end{abstract}

Index Terms - Accelerator magnets, high-luminosity large hadron collider (LHC) project, Nb3Sn $11 \mathrm{~T}$ dipole, superconducting magnets.

\section{INTRODUCTION}

$\mathrm{C}$ OLliMATORS are installed in the Large Hadron Collider (LHC) to safely intercept and absorb beam losses [1]. In order to cope with intensities, which are larger than nominal like in the High Luminosity LHC (HiLumi LHC) Project [2], additional collimators will be installed in the Dispersion Suppressor (DS) regions at the location of selected $14.3 \mathrm{~m}$ long, 8.3 T Nb-Ti LHC main bending dipoles (MBs) [3], [4]. Each concerned $\mathrm{MB}$ will be replaced by an $11 \mathrm{~T}$ Dipole Full Assembly (MBHFA) made of two shorter $11 \mathrm{~T}$ dipoles (MBH), symmetrically installed around the center of the replaced MB, and a bypass cryostat. These MBHs shall be compatible with the LHC lattice and main systems. The MBHFA will be connected in series with the MBs, and shall produce the same integrated field, $119 \mathrm{~T} \cdot \mathrm{m}$ at $11.85 \mathrm{kA}$ [5], [6].

Following tests carried out during heavy ions runs of LHC in the end of 2015 [7], [8], [9], and further to a revision of the project scope in June 2016 driven by cost considerations, it was decided to install (1) two additional collimators, one per beam on either side of interaction point 7 (IP7) for both proton and heavy-ion collimation losses, and (2) two additional collimators for heavy-ion secondary beams, one on either side of IP2 at the location of the connection cryostats in between the first MB and the first quadrupole, Q11, of the DS region next to the arc.

\section{DESIGN FEATURES OF THE 11T DiPOLE MAGNET}

The MBHFA is made of three cryo-assemblies, which will be installed and aligned independently: two of these each housing an $\mathrm{MBH}$ cold mass of 6.25-m length, and a bypass cryostat of 2.16-m length installed between them. The bypass cryostat ensures the continuity of the cryogenic and electrical circuits and comprises cold to warm transitions on one of the beam lines in order to create a room temperature vacuum sector sufficiently long to install the collimator equipped with jaws of $600-\mathrm{mm}$ active length. The overall length of the MBHFA is $15.66 \mathrm{~m}$, including the interconnects. Its integration with the collimators in the LHC is amply described in [10]. Fig. 1 shows a side and a front view of the MBHFA. The MBH has the same outside diameter as the $\mathrm{MB}$ in order to allow reusing, for the welding of the shrinking cylinder, the tooling recovered from the LHC construction era. The coil aperture is $60 \mathrm{~mm}$ and the inside diameter of the cold bore tube is $50 \mathrm{~mm}$, like in the MB.

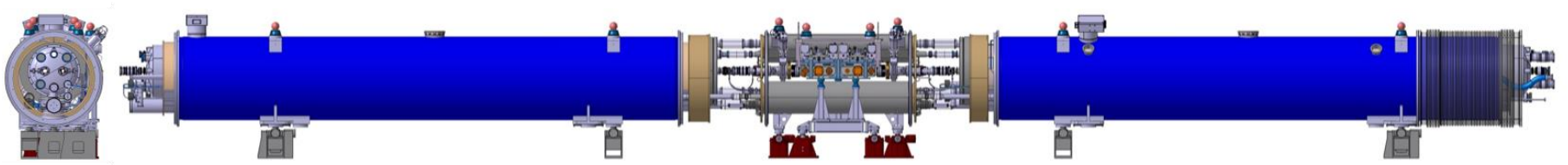

Fig. 1. Side view on the connection side with the diode container, and front view of the MBHFA with the collimator integrated in the middle, with an LMBHA on the left and an LMBHB on the right.

Manuscript received September 6, 2016.

F. Savary, M. Bajko, B. Bordini, L. Bottura, L. Fiscarelli, J. Fleiter, A. Foussat, S. Izquierdo Bermudez, F. Lackner, C. H. Löffler, J. C. Perez, H. Prin, R. Principe, D. Ramos, G. de Rijk, D. Smekens, S. Sequeira Tavares, and G. Willering are with the European Organization for Nuclear Research, CERN, 1211 Geneva, Switzerland (e-mail: Frederic.Savary@cern.ch).

A. V. Zlobin is with Fermi National Accelerator Laboratory, Batavia, IL 60510 USA. 
The main parameters of the MBH are given in Table I. As it will be installed in lieu of an MB, the MBH features a two-inone structure, however with separate collars as shown in Fig. 2, unlike the MB, which has common collars for the two apertures.

The cable for the first four MBHs will be made of Restacked Rod Process (RRP) conductor, whereas the cable for the two foreseen spare MBHs would be made of Powder in Tube (PIT) conductor. For the PIT route, a new cable cross section, hence a new coil cross section, were studied in order to avoid the excessive degradation of the critical current that was observed after the cabling of first batches of PIT conductor [11].

TABLE I

MaIN PARAMETERS OF THE 11 T Dipole Full ASSEMBly

\begin{tabular}{ll}
\hline \hline \multicolumn{1}{c}{ Quantity } & \multicolumn{1}{c}{ Value } \\
\hline Aperture & $60 \mathrm{~mm}$ \\
Cold mass outer diameter & $570 \mathrm{~mm}$ \\
Magnetic length & $5.307 \mathrm{~m}$ \\
Coil physical length, as per magnetic design & $5.417 \mathrm{~m}$ \\
Magnet physical length: active part & $5.785 \mathrm{~m}$ \\
Magnet physical length: cold mass & $6.252 \mathrm{~m}$ \\
Cold mass weight & $\sim 8$ tonne \\
Operating temperature & $1.9 \mathrm{~K}$ \\
Nominal operation current & $11.85 \mathrm{kA}$ \\
Bore field at nominal current & $11.23 \mathrm{~T}$ \\
Peak field at nominal current & $11.77 \mathrm{~T}$ \\
Load line margin & $20 \%$ \\
EM-force Fx/Fy per quadrant at $\mathrm{I}_{\text {nom }}$ & $6.22 / 3.16 \mathrm{MN} / \mathrm{m}$ \\
Stored energy/m at $I_{\text {nom }}$ & $0.9663 \mathrm{MJ} / \mathrm{m}$ \\
Differential inductance/m at $I_{\text {nom }}$ & $11.97 \mathrm{mH} / \mathrm{m}$ \\
Number of turns (inner/outer layer) & $56(22 / 34)$ \\
Cable bare width before reaction & $14.7 \mathrm{~mm}$ \\
Cable bare mid-thickness before reaction & $1.25 \mathrm{~mm}$ \\
Keystone angle, RRP/PIT & $0.79 \% / 0.50^{\circ}$ \\
Strand diameter & $0.700 \pm 0.003$ \\
Number of strands per cable & 40 \\
Cu to non-Cu ratio & $1.15 \pm 0.10$ \\
RRR, after reaction & 150 \\
Minimum strand critical current, $I_{\mathrm{c}}$, without self- & $438 \mathrm{~A}$ \\
field correction (12 T, 4.222 K) & $2560 \mathrm{~A}$ \\
Minimum strand current density, $J_{\mathrm{c}}(12 \mathrm{~T}, 4.222 \mathrm{~K})$ & $0.155 / 0.100 \mathrm{~mm}$ \\
Cable insulation thickness before/after reaction & \\
\hline \hline
\end{tabular}

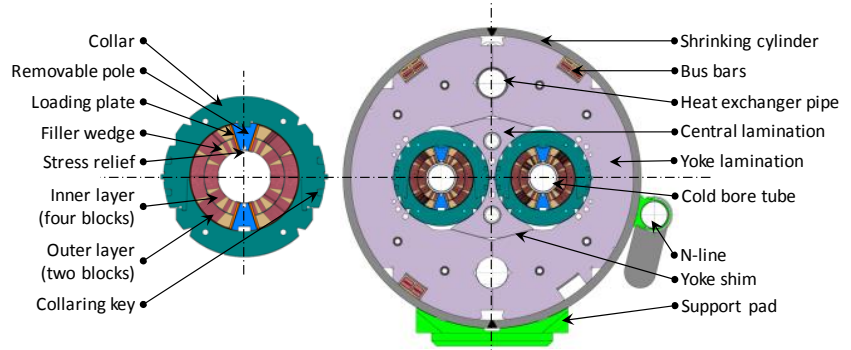

Fig. 2. Cross section of the collared coils (left) and of the MBH cold mass (right).

Allowable degradation within the limit of $5 \%$ was made possible by reducing the keystone angle from $0.79^{\circ}$ to $0.50^{\circ}$, keeping the same mid-thickness [12]. The corresponding increase of thickness at the thin edge $(37 \mu \mathrm{m})$ results in critical current degradation of $3 \%$ in average.

\section{DEVELOPMENT ACTIVITIES}

\section{A. Short Model Programme}

The fabrication of the short models started at FNAL in 2011, and later at CERN in 2013. There were three single aperture models, a mirror assembly, and a two-in-one model made at FNAL, and there were one single coil model, four single aperture models, and a two-in-one model made at CERN to date. The cross section of the different models made to date, at FNAL and at CERN, and their design features, are described in [13]. The results of the performance tests are extensively reported in [14], [15], [16], [17], [18]. The tests results of the last single aperture model made at CERN, MBHSP104, are currently being analysed and will be reported later. In this paper, we focus on the quench performance tests of the first two-in-one model made at CERN, MBHDP101, of which the cross section is almost identical to that of the final magnet shown in Fig. 2 (right). In this model, the bus bars, the heat exchanger tube, the cold mass support pads, and the yoke shim between the yoke laminations and the central laminations are not present. Also, the outside diameter of this model is larger, equal to $580 \mathrm{~mm}$. Beyond the models made to date, it is planned to produce a single aperture model, MBHSP105, with embedded quench heaters $(\mathrm{QH})$, i.e. impregnated with the coils in order to improve their efficiency thanks to closer contact with the coils, and hence a lower thermal resistance [19]. In addition to this, the gelling and post-curing after impregnation of the coils was done in a pressurized mould under $\sim 3$ bar in order to make sure the coil is free from pores and voids. Another model, MBHSP106, will be made with embedded QHs and inter-layer quench heaters (ILQH). These ILQH, which would complement the QH installed on the outside surface of the coil, should improve further the performance of the quench protection system, and furthermore add redundancy in the system, making it more robust. After these models, there will be four models made with the new cross section, optimized for PIT conductor. There will be three single aperture models and a two-in-one model made with PIT conductor. An additional single aperture model may be made with RRP conductor in order to verify that it is compatible with the new cross section.

\section{B. Quench Performance}

The quench performance of the first $\mathrm{MBH}$ single aperture models fabricated at CERN, namely MBHSP101, 102, and 103, has been extensively discussed in [18]. The collared coils used in the models MBHSP102 and 103 were removed from their single aperture structure, and assembled in a two-in-one structure. The training of this two-in-one model, MBHDP101, turned out to be very fast, as no quench occurred at the first ramp up to $11.87 \mathrm{kA}$. Then, the test had to be interrupted due to a joint quenching in the test station. When the cold tests were resumed, only two quenches were needed to exceed the ultimate field of $12 \mathrm{~T}$ [20]. Thereafter, a number of quenches were made to understand the magnet behaviour under high MIITs, as part of magnet protection studies. During these tests, $94 \%$ of the short sample limit was reached, concurring with the mechanical limit of the $\mathrm{MBH}$ structure. The quench performance of 
MBHDP101 is illustrated in Fig. 3 with the results of the single aperture models MBHSP102 and 103 for comparison. The better performance of the MBHDP101 is attributed to the fact that (1) the coils were trained when tested in the single aperture structure, and (2) the collaring parameters, in particular the thickness of the collaring shims allowing to tune the level of prestress in the coils, were optimized for the two-in-one structure. The collared coils were not dismounted, meaning that the collaring shims were not changed, when the collared coils were transferred in the two-in-one structure. However, the thickness of the shims installed between the collars and the yoke were increased by $50 \mu \mathrm{m}$ for the collared coils originally assembled in MBHSP102.

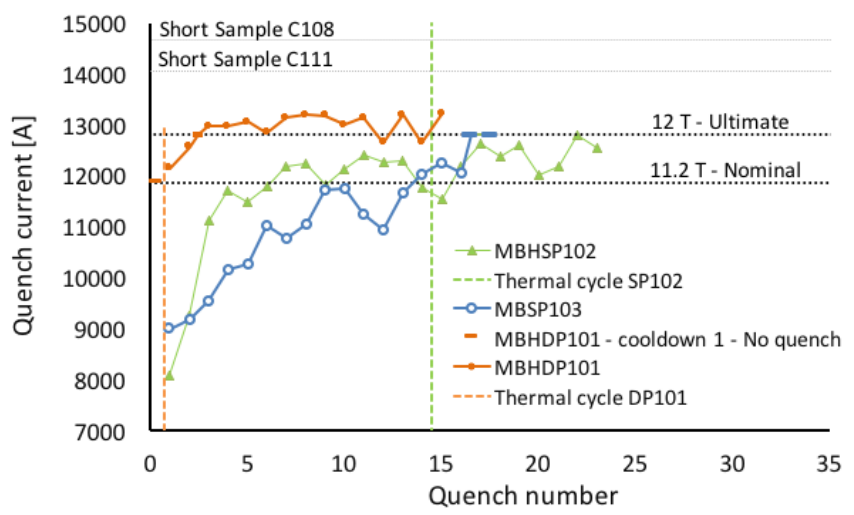

Fig. 3. Quench performance of MBHDP101, the first two-in-one model of the MBH made at CERN. It is made of the coils \#106 and \#108 of the model MBHSP102, and of the coils \#109 and \#111 of the model MBHSP103.

\section{FULL-SCALE PROTOTYPE}

\section{A. Description}

A full scale prototype, representative of the final magnet shown in Fig. 2, is under fabrication at CERN. The end cover on the side of the bypass cryostat had to be enlarged and made flat in order to create sufficient space for the integration of the collimator. On the other side, the end cover is strictly identical to the one of the MB for obvious reason of integration in LHC. On both sides, the fittings of (1) the M-lines providing passage for the bus bars through the MBHFA, (2) the X-line for the heat exchanger pipe, (3) the V-lines for the cold bore tubes, and (4) the instrumentation feed through systems (IFSs), have been made identical to those of the MBs.

The operation of the LHC requires that the magnets are equipped with instrumentation to monitor voltages at critical locations. Temperature is also measured at mid-length on the outside diameter of the yoke, and a cryo-resistance is attached to the end plate of the magnet. Finally, the QHs need cabling for their powering. The instrumentation and number of associated wires are listed in Table II. Seen the total number of wires needed, the installation of two standard IFS systems like those used in the MBs is necessary.

The manufacturing procedures and the tooling required for the fabrication of the coils are derived from the methods applied for the short models produced at an earlier stage of the project. The manufacturing procedures applied for the assembly of the coils with the collars, and for the construction of the cold mass are well mastered at CERN. Special attention was required for the fabrication of the long coils, especially for the reaction and impregnation processes, which are specific to $\mathrm{Nb}_{3} \mathrm{Sn}$. Completely new tooling had to be developed and manufactured on purpose for these critical operations; they are now available at CERN, and fully operational.

TABLE II

INSTRUMENTATION IN THE MBHFA

\begin{tabular}{lcc}
\hline \hline \multicolumn{1}{c}{ Description } & $\begin{array}{c}\text { Nb. of wires } \\
\text { LMBHA }\end{array}$ & $\begin{array}{c}\text { Nb. of wires } \\
\text { LMBHB }\end{array}$ \\
\hline V-Taps for joints and coils & 16 & 16 \\
V-Taps for trim circuit and diode & 8 & - \\
V-Taps for spool pieces & 4 & 2 \\
Quench heaters (4 circuits per aperture) & 16 & 16 \\
Thermometer & 4 & 4 \\
Cryo-resistance & 2 & 2 \\
Total & 50 & 40 \\
\hline \hline
\end{tabular}

There are 1 wire per V-Tap, 2 wires per $\mathrm{QH}, 4$ wires per thermometer, and 2 wires per cryo-resistance.

Three practice coils were wound, cured, and reacted, one with copper conductor and two with low grade (meaning not fully qualified) superconducting wire, in order to develop the procedures, train the technical staff, and commission the machines and tooling. Two of these coils were impregnated successfully. The first two full performance coils were wound and cured, and are now waiting for reaction and impregnation.

\section{B. Reaction Process}

The coils are reacted after winding in order to form the superconducting $\mathrm{Nb}_{3} \mathrm{Sn}$ compound. For this, they are put in a mould called the reaction fixture (RF), and the reaction is realized in a furnace operating under $\mathrm{Ar}$ atmosphere. The $\mathrm{RF}$ is sized taking into account the volumetric expansion of the cable during reaction, which is of the order of $1 \%$ in width (radial direction in the coil) and 3\% in thickness (azimuthal direction in the coil). The furnace is equipped with a retort housing the coil and RF assembly during reaction.

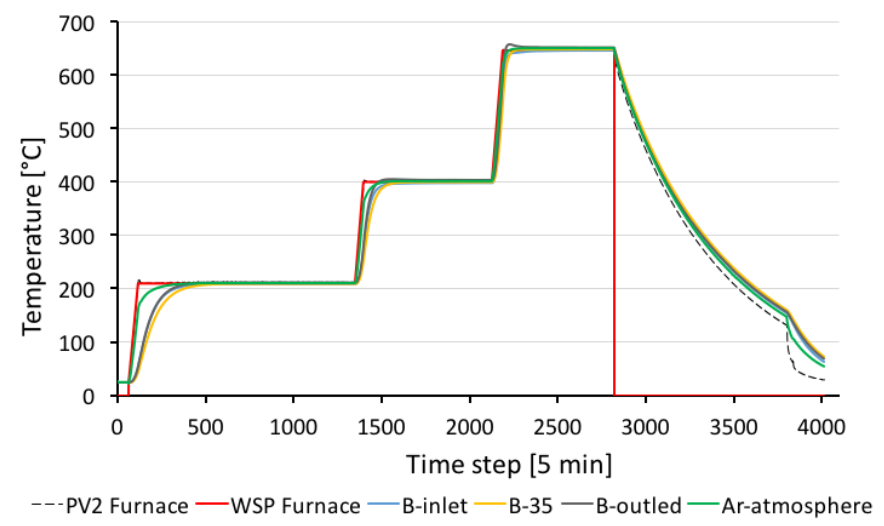

Fig. 4. Reaction cycle of the coil MBH_CR000002. WPS is the working set point, $\mathrm{PV} 2$ is the actual temperature of a heating zone, $\mathrm{B}$ is the temperature of a selection of points in the mould, and Ar-atmosphere is the temperature of the $\mathrm{Ar}$ atmosphere in the retort.

The reduction of oxygen is obtained by flushing the retort and the RF with Ar till the lowest achievable level of oxygen. The oxygen residual is measured by means of galvanic $\mathrm{ZrO}$ sensors. The reaction process requires several temperature 
plateaus up to $650^{\circ} \mathrm{C}$ during which the homogeneity of temperature in the coil shall be better than $\pm 3^{\circ} \mathrm{C}$. The development of the tooling and the optimization of the process are extensively described in [21]. An example of reaction cycle including the set points for the temperature and the temperature measured with thermocouples of K-type at different locations in the reaction fixture are shown in Fig. 4. In total, there are 30 thermocouples.

\section{Impregnation}

The coils are impregnated after they have been reacted with an anhydride epoxy system CTD-101K from the Composite Technology Development company. This resin system has a pot life of 20 hours, and a low viscosity of the order of $100 \mathrm{cP}$ at $60^{\circ} \mathrm{C}$, which has been choosen as processing temperature.

The cable insulation system comprises a first layer of $80 \mu \mathrm{m}$ thick Mica tape rolled around the cable in a $\mathrm{C}$-shaped profile and a $70 \mu \mathrm{m}$ thick layer of S2-glass braided over the Mica layer. This insulation will reduce down to $100 \mu \mathrm{m}$ after reaction. The S2-glass layer comprises 32 threads of over braided bundles of 9 yarns per thread. The yarn is made of AGY S- $2^{\circledR} 11$ TEX 636, and there are 12 threads over $20 \mathrm{~mm}$. The Mica tape is COGEBI FIROX $^{\circledR}$, which is made of phlogopite Mica glued on an E-glass backing strip. The Mica tape has a dielectric strength of $800 \mathrm{~V}$. The coil insulation system will be tested by means of a capacitive discharge at $4.7 \mathrm{kV}$ corresponding to $85 \mathrm{~V} /$ turn on the finished coils, prior to their assembly with the collars. Electrical tests were carried out on practice coils up to $7 \mathrm{kV}$ before observing signs of degradation.

The preparation for impregnation includes the realization of $\mathrm{Nb}_{3} \mathrm{Sn}$ to $\mathrm{Nb}$ - $\mathrm{Ti}$ joints at the coil terminals. The extensions made of $\mathrm{Nb}$-Ti conductor are stabilized with a copper strip of $1.9 \mathrm{~mm}$ thickness. They will allow shaping safely the cable for the joints between the poles and the magnet apertures, and with the bus bars, during the subsequent stages of the construction, which would not be possible with $\mathrm{Nb}_{3} \mathrm{Sn}$ that is largely embrittled after reaction. The metallic saddles at the ends of the coils are replaced by $\mathrm{G} 11$ pieces in order to avoid electrical faults in this region, especially with the $\mathrm{Nb}_{3} \mathrm{Sn}$ to $\mathrm{Nb}$ - $\mathrm{Ti}$ joints, which are embedded in the these saddles. The QHs are placed onto the outside surface of the coil, and an $80 \mu \mathrm{m}$ thick wrap made of glass fiber is placed onto the $\mathrm{QH}$.

The impregnation system is equipped with a mixing reservoir and an outlflow reservoir, which can both be evacuated and pressurized up to 5 bar. The vacuum chamber housing the impregnation mould during the process is tilted by $18^{\circ}$. In order to achieve a good level of vacuum in the mould prior to injection, the mould is heated to $110^{\circ} \mathrm{C}$ and flushed several times with nitrogen. It is then cooled down to $60^{\circ} \mathrm{C}$, and evacuated, for resin injection. Levels of vacuum comprised between $2 \times 10^{-1}$ and $5 \times 10^{-2}$ mbar in the mixing and outlflow reservoirs, and between $5 \times 10^{-2}$ and $7 \times 10^{-3}$ mbar in the vacuum chamber are considered satisfactory to start the resin injection. The mandrel of the impregnation mould features two main longitudinal feeder channels, which connect to the mid-plane of the coil via a series of $0.1 \mathrm{~mm}$ deep and $12 \mathrm{~mm}$ wide transversal channels, every $5 \mathrm{~mm}$, allowing for a uniform distribution of resin along the coil. It takes 4 to 5 hours to fill the coil completely. After injection, the resin is heated up to $110^{\circ} \mathrm{C}$ for 5 hours for gelling, and up to $125^{\circ} \mathrm{C}$ for 16 hours for postcuring. Prior to launching the curing cycle, the impregnation mould is pressurized to 3 bar in order to ensure absence of pores or voids. Fig. 5 shows the temperature measurements during a curing cycle of the first superconducting practice coil.

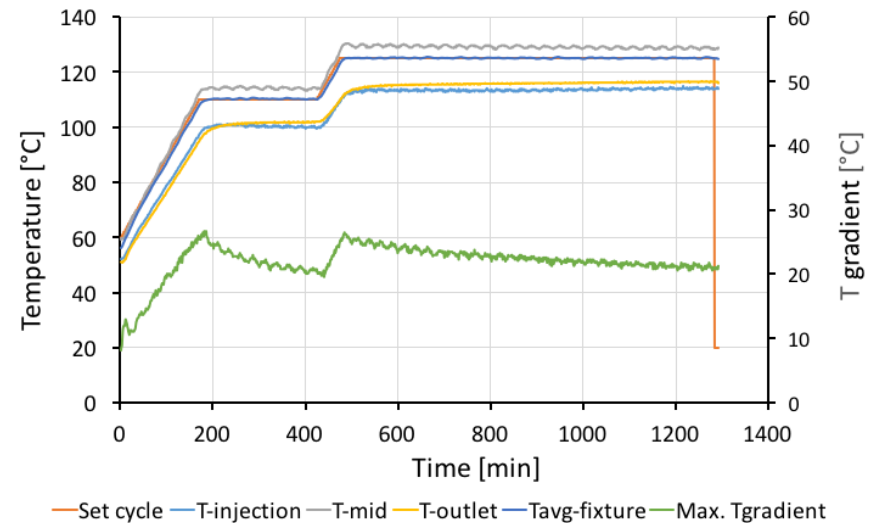

Fig. 5. Curing cycle of the coil MBH_CR000001. Tgradient indicates the difference of temperature between the highest and the lowest temperature measured in the mould.

\section{Plan TOWARdS PRODUCTION FOR LS2}

The fabrication and testing of the prototype described in this paper is a mandatory step prior to start the fabrication of the four MBHs needed for installation during the years 2019-2020. The tests of this prototype are expected to occur in the last quarter of 2017. The fabrication of the coils for the series magnets should start not later then November 2017 to meet the schedule requirements for LS2. Every MBH will be tested independently, and an MBHFA will be tested on the surface as soon as two MBHs are available in order to verify the integration features and performance of the $\mathrm{MBHs}$ in fully representative conditions.

The fabrication of the second prototype with PIT conductor will start after the four $\mathrm{MBHs}$ for installation are ready available, and the fabrication of the two spare $\mathrm{MBH}$ s will start after validation of the PIT route with the prototype.

\section{CONCLUSION}

Although the development programme of the $\mathrm{MBH}$ for the HiLumi LHC project is not finished, an important milestone was reached with the successful tests of the first two-in-one model of 2-m length fabricated at CERN. The most critical tooling for the 5.5-m-long coils, the reaction furnace and the impregnation system, are now available at CERN and fully operational following the fabrication of the first full-length superconducting coils. The fabrication schedule of the MBHs needed for LS2 is tight; however, satisfactory solutions were found for all the technical challenges and there is no showstopper for the series production. The fabrication of the first prototype, and the completion of the cold tests in the last quarter of 2017 are now the most important milestone of the $11 \mathrm{~T}$ dipole project at CERN. 


\section{REFERENCES}

[1] R. W. Assmann et al., "The final collimation system for the LHC," in Proc. EPAC, 2006, pp. 986-988.

[2] L. Rossi, "LHC upgrade plans: Options and strategy," in Proc. IPAC, 2011, pp. 908-912.

[3] J. M. Jowett et al., "LHC collimation review," CERN, Geneva, Switzerland, May 30, 2013.

[4] B. Roderick et al., "Conceptual design of IR collimation," CERN, Geneva, Switzerland. [Online]. Available: https://cds.cern.ch/record/ 1972595/files/CERN-ACC-2014-0293.pdf.

[5] G. de Rijk et al., "11 Tesla $\mathrm{Nb}_{3}$ Sn dipoles for Phase II collimation in the Large Hadron Collider," CERN, Geneva, Switzerland, sLHC Project Note 0019, 2010.

[6] L. Rossi et al., "Advanced accelerator magnets for upgrading the LHC," IEEE Trans. Appl. Supercond., vol. 22, no.3, Jun.2012, Art.no.4002008.

[7] P. D. Hermes et al., LHC Heavy-Ion Collimation Quench Test at $6.37 \mathrm{Z}$ TeV, CERN-ACC-Note-2016-0031 MD, March 17, 2016.

[8] J. Jowett et al., Bound-Free Pair Production in LHC Pb-Pb Operation at 6.37 Z TeV Per Beam, Proceedings of IPAC2016, Busan, Korea 2016, DOI:10.18429/JACoW-IPAC2016-TUPMW028.

[9] C. Bahamonde Castro et al., Power Deposition in LHC Magnets due to Bound-Free Pair Production in the Experimental Insertions, Proceedings of IPAC2016, Busan, Korea 2016, DOI:10.18429/JACoW-IPAC2016TUPMW006.

[10] D. Ramos et al., "Integration of the $11 \mathrm{~T} \mathrm{Nb}_{3}$ Sn dipoles and collimators in the LHC," IEEE Trans. Appl. Supercond., vol. 26, no. 4, Jun. 2016, Art. no. 3800105 .

[11] J. Fleiter et al., "Optimization of $\mathrm{Nb}_{3} \mathrm{Sn}$ Rutherford cables geometry for the High Luminosity LHC," in This conference, ASC 2016 Denver.

[12] E. Nilsson et al., "Design Optimization of the $\mathrm{Nb}_{3} \mathrm{Sn} 11 \mathrm{~T}$ Dipole for the High Luminosity LHC," in This conference, ASC 2016 Denver.

[13] F. Savary et al., "The 11 T Dipole for HL-LHC: Status and Plan," IEEE Trans. Appl. Supercond., vol. 26, no. 4, Jun. 2016, Art. no. 4005305.

[14] A. V. Zlobin et al., "Development and test of a single-aperture $11 \mathrm{~T} \mathrm{Nb}_{3} \mathrm{Sn}$ demonstrator dipole for LHC upgrades," IEEE Trans. Appl. Supercond., vol. 23, no. 3, Jun. 2013, Art. no. 4000904.

[15] A. V. Zlobin et al., "Quench performance of a $1 \mathrm{~m}$ long single-aperture $11 \mathrm{~T} \mathrm{Nb}_{3} \mathrm{Sn}$ dipole model for LHC upgrades," IEEE Trans. Appl. Supercond., vol. 24, no. 3, Jun. 2014, Art. no. 4000305.

[16] A. V. Zlobin et al., "Status of $11 \mathrm{~T}$ 2-in-1 Nb3Sn dipole development for LHC," presented at the International Particle Accelerator Conference, Dresden, Germany, 2014, Paper WEPRI097.

[17] A. V. Zlobin et al., "Quench performance of the first twin-aperture $11 \mathrm{~T}$ dipole for LHC upgrades," presented at the International Particle Accelerator Conference, Richmond, VA, USA, 2015, Paper WEPTY040.

[18] G. P. Willering et al., "Cold powering tests of $11 \mathrm{~T} \mathrm{Nb}_{3}$ Sn dipole models for LHC upgrades at CERN," IEEE Trans. Appl. Supercond., vol. 26, no. 4, Jun. 2016

[19] S. Izquierdo Bermudez et al., "Quench protection studies of the $11 \mathrm{~T}$ $\mathrm{Nb}_{3} \mathrm{Sn}$ dipole for the LHC upgrade," IEEE Trans. Appl. Supercond., vol. 26, no. 4, 2016.

[20] G. Willering et al.; "Cold powering performance of the first 2 meter Nb3Sn DS11T twin aperture model magnet at CERN," in This conference, ASC 2016 Denver.

[21] F. Lackner et al., "Analysis of temperature uniformity during heat treatment of $\mathrm{Nb}_{3} \mathrm{Sn}$ coils for the High Luminosity LHC superconducting magnets," IEEE Trans. Appl. Supercond., vol. 26, no. 4, Jun. 2016 